\title{
CARACTERISTICAS DE LA FLOTA PESQUERA COMERCIAL DE IQUITOS
}

\author{
J.S. Tello* \\ V.H. M ontreuil *
}

\section{ANTECEDENTES}

L a pesca en la A mazonía peruana, ocupa un lugar importante dentro de las actividades que sustentan la economía en la región. La principal fuente de abastecimiento de proteína animal de las poblaciones asentadas a lo largo de los numerosos ríos existentes, es el pescado y todos los desembarques provienen tanto de la flota comercial como la de sostenimiento.

La pesca de subsistencia se realiza a nivel familiar, empleando para ello embarcaciones pequeñas (canoas) y artes de pesca bastante sencillas (flechas, anzuel os y atarraya). La flota comercial utiliza embarcaciones de mayor tamaño y están equipados con cajas isotérmicas y artes de pesca más complejos (redes).

Chaprnan (1981), realizó la evaluación de capturas en el río A mazonas de la pesca de sostenimiento. Para este trabajo, empleó el método del conteo aéreo de canoas y las encuestas en villorios, en los ríos denominados grandes (U cayali y Marañón), medianos (Tapiche y Puinahua) y pequeños (Juanache y M aquía). Los resultados de este trabajo determinaron una captura aproximada de 342,000 t. para la cuenca amazónica del Perú por debajo de los $240 \mathrm{~m}$. (alrededor de $520,300 \mathrm{~km} 2$ ). Esta estimación no incluye la producción de la flota comercial. El mismo autor, utilizando las encuestas hechas por el IMARPE-Iquitos, en 1978, a las embarcaciones que conforman esta última, determina una captura de $871 \mathrm{t}$. y 2,376 t. para el período de creciente y vaciante, respectivamente. A dicionalmente, describe, en forma general, las características de este tipo de embarcaciones pesqueras.

\section{OBJETIVO GENERAL}

Contribuir al ordenamiento de la pesquería en la A mazonía peruana, con el fin de realizar la explotación sostenida del recurso.

* Investigadores Dirección de Recursos Hidrobiológicos, Instituto de Investigaciones de la Amazonía Peruana -IIAP. Av. Abelardo Quiñones Km. 2.5 Apartado 784. Iquitos- Perú. 


\section{OBJETIVO ESPECIFICO}

Presentar una descripción detallada de la flota pesquera comercial de Iquitos.

\section{LINEAS DE INVESTIGACION}

Este trabajo forma parte de las actividades programadas por el Proyecto "Evaluación de Recursos Pesqueros" del Instituto de Investigaciones de la A mazonía Peruana (IIAP). Se llevó a cabo en el puerto de Belén, lugar de mayor importancia en el desembarque de pescado en la zona de Iquitos. En 1989, se encuestaron a 20 embarcaciones (50\% del total de la flota) con registro y concesión de pesca, cuyo radio de acción abarca las cuencas de los ríos U cayali y M arañón.

Los formularios de las encuestas se diseñaron y modificaron tomando como base los formatos utilizados por FA O (1968) para embarcaciones científicas o buques de investigación.

Para el cálculo de la inversión inicial y costos de operación, se tomó en cuenta el trabajo de Cruz (1990).

Se tomaron fotografías para utilizarlas como modelos en los dibujos de los diferentes tipos de embarcaciones pesqueras y para el procesamiento de la información, así como para los gráficos se empleó una computadora. Las flotas comerciales que operan en Pucallpa, Y urimaguas y Caballococha no fueron consideradas en este estudio.

\section{LOGROS}

\section{EMBARCACIONES}

En el Cuadro 1, se observa que el material de construcción empleado por el $90 \%$ de la muestra fue en su totalidad madera. Una de ellas (1\%), estaba construida enteramente de hierro, otras presentaban el casco de hierro y las demás, estructuras de madera. L as especies forestales más frecuentes para este fin son: canela moena (Endlicheria anomala), itauba (M ezilaurus itauba), moena amarilla (Aniba sp), cedro (Cedrela sp), lagarto caspi (Potrium apiculatum), anacaspi (Apuleia sp) y catahua (Hura crepitans). 


\section{Cuadro 1}

\section{Dimensiones principales y material del Casco - Estructura}

\begin{tabular}{|c|c|c|c|c|c|c|}
\hline 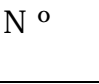 & $\begin{array}{l}\text { E dad } \\
\text { A ños }\end{array}$ & $\begin{array}{c}\text { Escolar } \\
(\mathrm{m})\end{array}$ & $\begin{array}{l}\text { M anga } \\
(\mathrm{m})\end{array}$ & $\begin{array}{c}\text { Puntal } \\
(\mathrm{m})\end{array}$ & $\begin{array}{c}\text { Calado } \\
(\mathrm{m})\end{array}$ & M aterial \\
\hline 1 & 20 & 16.0 & 6.0 & 3.0 & 2.10 & MADERA \\
\hline 2 & 20 & 17.0 & 3.8 & 3.0 & 1.20 & MADERA \\
\hline 3 & 10 & 17.0 & 3.0 & 6.0 & 1.20 & MADERA \\
\hline 4 & 5 & 15.0 & 4.0 & 3.5 & 2.10 & MADERA \\
\hline 5 & 5 & 17.8 & 3.5 & 4.8 & 1.20 & MADERA \\
\hline 6 & 5 & 17.0 & 4.0 & 3.0 & 2.10 & MADERA \\
\hline 7 & 4 & 21.0 & 6.0 & 6.0 & 1.40 & FIERRO \\
\hline 8 & 10 & 15.0 & 3.5 & 4.0 & 1.20 & MADERA \\
\hline 9 & 5 & 19.0 & 3.7 & 1.9 & 0.90 & MADERA \\
\hline 10 & 5 & 17.0 & 4.0 & 3.5 & 2.10 & MADERA \\
\hline 11 & 2 & 20.0 & 4.0 & 3.0 & 2.10 & MADERA \\
\hline 12 & 6 & 18.0 & 3.8 & 3.5 & 0.90 & MADERA \\
\hline 13 & 3 & 16.0 & 4.0 & 5.5 & 1.00 & $\begin{array}{l}\text { FIERRO-CASCO } \\
\text { MADERA-ESTRUCT. }\end{array}$ \\
\hline 14 & 3 & 20.9 & 4.0 & 3.5 & 1.60 & MADERA \\
\hline 15 & 6 & 17.8 & 3.8 & 4.5 & 1.50 & MADERA \\
\hline 16 & 4 & 16.0 & 3.0 & 2.8 & 1.50 & MADERA \\
\hline 17 & 5 & 26.0 & 5.0 & 5.5 & 1.80 & MADERA \\
\hline 18 & 5 & 13.0 & 3.0 & 3.5 & 0.90 & MADERA \\
\hline 19 & 6 & 14.0 & 3.0 & 3.0 & 0.90 & MADERA \\
\hline 20 & 0.5 & 22.0 & 5.0 & 3.5 & 1.50 & MADERA \\
\hline Prom. & 6 & 17.6 & 4.8 & 3.8 & 1.50 & \\
\hline Rango & $0.5-20$ & $13-26$ & $3-6$ & $1.9-6$ & $0.9-2.1$ & \\
\hline
\end{tabular}

En el mismo cuadro, vemos que la eslora promedio de una embarcación comercial es de $17 \mathrm{~m}$., con un rango que oscila entre los 13 y $26 \mathrm{~m}$. La manga promedio es de $4 \mathrm{~m}$., con un rango de $36 \mathrm{~m}$. La altura o puntal, considerado desde la plantilla hasta la altura mayor, fue de $4 \mathrm{~m}$. en promedio, con un rango de $0,9 \mathrm{~m}$. a2. $1 \mathrm{~m}$.

L a edad promedio de las embarcaciones encuestadas fue de 6 años, con un rango de 6 meses a 20 años. El $6 \%$ de ellas fueron construidas en Brasil ( $M$ anaos) y el resto en Iquitos y Pucallpa (Perú). 


\section{MOTORES Y EQUIPOS AUXILIARES}

Todas las embarcaciones encuestadas utilizan como sistema de propulsión principal, motores de centro con una potencia promedio de $60 \mathrm{HP}$ y con un rango de 20 a $270 \mathrm{HP}$. Para las faenas de pesca, se emplean botes auxiliares con longitud promedio de $8 \mathrm{~m}$. y un rango d 6-12 m., cuya fuerza motriz consiste en motores fuera de borda de 5 a $55 \mathrm{HP}$ y/o motores estacionarios de 3 a $12 \mathrm{HP}$ adaptados con una cola larga. Van acompañados por canoas cuya longitud promedio es de $5 \mathrm{~m}$., con un rango de 4-6 $\mathrm{m}$.

Todos los botes muestreados contaban con motobomba, generador de corriente eléctrica, faro y sondaleja. Sólo una de ellas presentaba equipo básico de seguridad: chaleco salvavidas, extinguidores, pistola de señales y botiquín de primeros auxilios.

\section{ARTES DE PESCA}

La flota pesquera comercial de Iquitos emplea con mayor frecuencia las redes tipo hondera (56\% del total de muestra), agallera (42\%) y arrastre (2\%). Dentro de las primeras, se observa la presencia de redes con longitud promedio de $100 \mathrm{~m}$., con un rango de $83-150 \mathrm{~m}$. La altura promedio determinada fue de 30 $\mathrm{m}$. y con un rango de $27-33 \mathrm{~m}$. En cuanto al tamaño de malla, se encontraron redes de $2 "$ que representaron el 78\% del total de la encuesta, el 17\% para las redes de $13 / 4^{\prime}$ y el $5 \%$ para las artes de pesca de 1 1/2".

En el caso de las redes agalleras, la longitud promedio fue de $70 \mathrm{~m}$. y la altura de $8 \mathrm{~m}$. El tamaño de malla varía desde 4' hasta 12'. Sólo una de las embarcaciones muestreadas llevaba una red tipo arrastre de $21 / 2 "$. El hilo nylon utilizado para la confección de las redes es del tipo polifilamento, cuyo número varía desde el 6 hasta el 240.

\section{ARTES DE PESCA Y CAPTURA COMERCIAL}

La flota comercial pesca durante todo el año y las zonas de mayor importancia se encuentran en las cuencas de los ríos U cayali y Marañón. La autonomía de pesca de las embarcaciones varía entre 20-30 días. El número promedio de viajes anuales por embarcación fue de 13, con un rango de 12-24. En la época de vaciante de los ríos, la duración de una faena o salida de pesca es de 12 días en promedio, con un rango de 6-15 días por embarcación. En creciente se incrementa 26 días en promedio, con un rango de 20-30 días. En cuanto a los días viajando, se determinó un promedio de 6, y con un rango de 312 días. Los días pescando, tuvieron un promedio de 5 y un rango de 3-10 días. 
L os desembarques de pescado de la flota comercial que opera en Iquitos se hacen al estado fresco, utilizando cajas isotérmicas de doble pared, cuyo espacio entre ellas es llenado totalmente con cascarilla de arroz para evitar la difusión de calor. Tienen una longitud promedio de $7 \mathrm{~m}$., con un rango de 5-12 m., la anchura en promedio es de $2.4 \mathrm{~m}$., con un rango de $1.74 \mathrm{~m}$. La altura en promedio fue de $1.7 \mathrm{~m}$., variando en un rango de 1-3 m., lo que nos da un volumen de $28 \mathrm{~m}^{3}$ en promedio y con un rango de 18 a $55 \mathrm{~m}^{3}$. La capacidad promedio de carga fue de $10 \mathrm{t}$, con un rango de 6-20 t. El número de planchas de hielo varía desde 300 hasta 1,200 y con un promedio de 663 por embarcación.

En el cuadro 2, se observa que las capturas de pescado fresco fueron significativas durante los años 1984-1985, sufriendo un decremento en 1986 para repuntar nuevamente en los años posteriores. Esta caída del volumen desembarcado, coincide con el mayor número de embarcaciones registrado en los últimos años.

\section{Cuadro 2}

Desembarque de pescado fresco en Iquitos.

\begin{tabular}{lrrrrrr}
\hline Número de & 1984 & 1985 & 1986 & 1987 & 1988 & 1989 \\
E mbarcaciones & 93 & 103 & 111 & 110 & 86 & 40 \\
\hline Captura total (1) & 3,009 & 3,429 & 2,932 & 3,169 & 3,500 & 3,811 \\
\hline
\end{tabular}

Fuente: Dirección Regional de Pesquería - L oreto.

El personal embarcado, hace las veces de tripulante y pescador. EI número promedio de pescadores por embarcación es de 9 y con un rango de 6-14. El $60 \%$ del personal encuestado trabaja bajo la modalidad de permanente, el resto lo hace como eventual. La repartición de utilidades provenientes de la comercialización de la captura se realiza luego de haber deducido los costos operativos de la faena de pesca y es en la proporción de $50 \%$ para el dueño de la embarcación y el saldo se reparte equitativamente entre todos los miembros de la tripulación.

En la composición de la captura en los últimos años se observa que el boquichico (Prochilodus nigricans) alcanza los mayores porcentajes $(24.4 \%)$ en los desembarques al estado fresco, seguido por yahuarachi (Potamorhina latior $16 \%$ ), ractacara (Curimata sp) - 7. $1 \%$ ), palometa (M ylossoma sp - 6.7\%) y dorado (Brachyplatystoma filamentosum) con el 5.9\%. En el cuadro 3, se incluye una lista de las especies que sustentan la pesquería en la A mazonía peruana. 


\section{Cuadro 3}

\section{Lista de las especies que sustentan la pesqueria en la Amazonía Peruana}

NOMBRE CIENTIFICO

Osteoglossum bicirrhosum

Arapaima gigas

Brycon sp.

Colossoma macropomum

Mylossoma sp.

Piaractus brachypomus

Triportheus sp.

Anodus elongatus

Prochilodus nigricans

Semaprochilodus sp.

Curimata sp.

Potamorhina altamazónica

Potamorhina latior

Psectrogaster sp.

Leporinus sp.

Schizodon fasciatus

Brachyplatystoma filamentosum

Pseudoplatystoma fasciatum

H ypophthalmus sp.

Pterygoplichthys multiradiatus

Plagioscion sp.
NOMBRE VULGAR

A rahuana

Paiche

Sábalo

Gamitana

Palometa

Paco

Sardina

Y ulilla

Boquichico

Y araquí

Ractacara

Llambina

Y ahuarachi

Chio-chio

Lisa

Lisa

Dorado

Doncella

M aparate

Carachama

Corvina

En el cuadro 4, se han agrupado las embarcaciones según el tamaño y sus características para facilitar el análisis económico y sus dibujos. Según este cuadro, el $57.5 \%$ de la flota comercial de Iquitos lo conforman las consideradas pequeñas, seguida de las grandes con el $27.5 \%$ y por último, las medianas con el $1 \%$ del total de la muestra. 


\section{Cuadro 4}

\section{C aracterísticas de las embarcaciones para el cálculo económico.}

\begin{tabular}{|c|c|c|c|c|c|c|c|c|c|c|}
\hline Tipo & \multirow[t]{2}{*}{$\begin{array}{l}\text { M otor } \\
\text { Princip. } \\
\text { (hp) }\end{array}$} & \multirow[t]{2}{*}{$\begin{array}{l}\text { Botes } \\
\text { Aux. }\end{array}$} & \multirow[t]{2}{*}{$\begin{array}{l}\text { Artes } \\
\text { Pesca }\end{array}$} & $\begin{array}{l}\text { M otor } \\
\text { A ux. }\end{array}$ & $\begin{array}{c}\text { Petróleo } \\
\text { (gal.) }\end{array}$ & \multirow[t]{2}{*}{$\begin{array}{l}\text { Gasolina } \\
\text { (gal.) }\end{array}$} & \multicolumn{2}{|c|}{\begin{tabular}{l}
\multicolumn{2}{c}{ A ceite } \\
Lubri. Caster \\
(gal.) (gal)
\end{tabular}} & \multicolumn{2}{|c|}{$\begin{array}{l}\text { Cap. de barras } \\
\text { Bodega de hielo } \\
\text { (t) }\end{array}$} \\
\hline & & & & $M / B^{*}$ & & & & & & \\
\hline Pequeña & 20 & 1 & 2 & $\begin{array}{l}\text { Gener. } \\
\text { F/B** } \\
M / B\end{array}$ & 200 & 25 & 0.5 & 8 & 8 & 400 \\
\hline M ediana & 50 & 2 & 4 & $\begin{array}{l}\text { Gener. } \\
\mathrm{P} / \mathrm{B} \\
\mathrm{M} / \mathrm{B}\end{array}$ & 400 & 50 & 1 & 15 & 12 & 600 \\
\hline Grande & $>50$ & 3 & 6 & Gener. & 600 & 100 & 2 & 20 & 25 & 1.000 \\
\hline Mot & obomba & & & & & & & & & \\
\hline ** $\quad$ M ot & or fuera $\mathrm{de}$ & borda & & & & & & & & \\
\hline $\begin{array}{r}\text { Deprecia } \\
\text { An }\end{array}$ & $\begin{array}{l}\text { ción (\%) } \\
\text { ual }\end{array}$ & & & $\begin{array}{l}\text { Unidades } \\
\text { Pequeñas }\end{array}$ & & $\begin{array}{l}\text { Unidades } \\
\text { Medianas }\end{array}$ & & & $\begin{array}{l}\text { idades } \\
\text { andes }\end{array}$ & \\
\hline Embarca & & & & 10 & & 10 & & & 10 & \\
\hline Botes aux & iliares & & & 10 & & 10 & & & 10 & \\
\hline M otor pri & ncipal & & & 10 & & 10 & & & 10 & \\
\hline M otor au & kiliar & & & 20 & & 20 & & & 20 & \\
\hline A rtes de & esca & & & 20 & & 20 & & & 20 & \\
\hline Caja isote & rmica & & & 100 & & 100 & & & 100 & \\
\hline
\end{tabular}

\section{CARACTERÍSTICAS ECONÓMICAS}

En el cuadro 5, se incluye el factor económico de cada tipo de embarcación, vale decir, la inversión en bienes de capital, en donde se considera lo más importante en cuanto a este rubro, como son: embarcación, motor principal y auxiliar, artes de pesca, etc. También se presentan los costos operativos, tales como: depreciación, combustible, lubricante, hielo y por último los ingresos anual es correspondientes.

Se ha determinado a través del análisis económico que las embarcaciones pequeñas son las más rentables, seguidas de las mayores y en último lugar las medianas. 


\section{Cuadro 5 \\ Calculo de los ingresos}

CONSIDERANDO: № PROMEDIO DE VIAJES/AÑO: 10

CAPACIDAD DE BODEGA

EMBARCACIONES PEQUEÑAS : $8 \mathrm{t}$.

EMBARCACIONES MEDIANAS : $12 \mathrm{t}$.

EMBARCACIONES GRANDES : $25 \mathrm{t}$.

PRECIO/KG. : USA \$ 1.00

\begin{tabular}{lcc}
\hline Embarcaciones & Volumen total & Ingreso (USA \$)/ \\
& A nual $(\mathrm{t})$ & A ño \\
\hline Pequeñas & 80 & 80,000 \\
M edianas & 120 & 120,000 \\
Grandes & 250 & 250,000 \\
\hline
\end{tabular}

\section{Cuadro 5}

Unidades E conómicas de Pesca (UE P)

\begin{tabular}{|c|c|c|c|c|c|c|}
\hline \multirow[b]{2}{*}{$\begin{array}{l}\text { Inversión en bienes } \\
\text { de capital }\end{array}$} & \multicolumn{2}{|c|}{ U nid. Pequeñas } & \multicolumn{2}{|c|}{ Unid. M edianas } & \multicolumn{2}{|c|}{ Unid. Grandes } \\
\hline & US\$ & $\%$ & US\$ & $\%$ & US\$ & $\%$ \\
\hline $\begin{array}{l}\text { Embarcación } \\
\text { Botes auxiliares } \\
\text { Motor principal } \\
\text { Motor auxiliar } \\
\text { A rtes de pesca } \\
\text { Caja isotérmica }\end{array}$ & $\begin{array}{l}2500 \\
150 \\
10000 \\
3500 \\
500 \\
150\end{array}$ & $\begin{array}{l}14.9 \\
0.9 \\
59.5 \\
20.8 \\
3.0 \\
0.9\end{array}$ & $\begin{array}{l}5000 \\
300 \\
16000 \\
3500 \\
1000 \\
200\end{array}$ & $\begin{array}{l}19.2 \\
1.2 \\
61.5 \\
13.5 \\
3.8 \\
0.8\end{array}$ & $\begin{array}{l}15000 \\
450 \\
35000 \\
3500 \\
1500 \\
250\end{array}$ & $\begin{array}{l}26.9 \\
0.8 \\
62.8 \\
6.3 \\
2.7 \\
0.5\end{array}$ \\
\hline $\begin{array}{l}\text { TOTAL } \\
\text { COSTOS OPERATIVOS } \\
\text { (US \$ANO) } \\
\end{array}$ & 16.800 & 100 & 26000 & 100 & 55700 & 100 \\
\hline $\begin{array}{l}\text { Depreciación } \\
\text { Combustibles y } \\
\text { Lubricantes } \\
\text { Hielo } \\
\text { Otros (Licencia, repintado } \\
\text { víveres, etc) }\end{array}$ & $\begin{array}{l}2215 \\
4356 \\
13000 \\
3000\end{array}$ & $\begin{array}{l}12.6 \\
\\
24.8 \\
45.5 \\
\\
17.1\end{array}$ & $\begin{array}{l}32.30 \\
8620 \\
12000 \\
4000\end{array}$ & $\begin{array}{l}11.6 \\
30.9 \\
43.0 \\
14.5\end{array}$ & $\begin{array}{l}6295 \\
12000 \\
20000 \\
6000\end{array}$ & $\begin{array}{l}14.3 \\
\\
27.0 \\
45.1 \\
13.6\end{array}$ \\
\hline TOTAL & 17571 & 100.0 & 27850 & 100.0 & 44295 & 100.0 \\
\hline $\begin{array}{l}\text { INGRESO/AÑO } \\
\text { UTILIDAD BRUTA }\end{array}$ & & $\begin{array}{l}000 \\
429\end{array}$ & & $\begin{array}{l}000 \\
150\end{array}$ & & \\
\hline $\begin{array}{l}\text { RENTABILIDAD (1) } \\
\text { (Indice) (\%) }\end{array}$ & & 371 & & 54 & & \\
\hline
\end{tabular}




$$
\begin{gathered}
I=\frac{\text { UTILIDAD BRUTA } x}{100} \\
\text { INVERSION }
\end{gathered}
$$

\section{PROBLEMAS}

De las embarcaciones encuestadas, el $80 \%$ se encontraban registradas en la Capitanía de Puerto, las restantes no contaban con el registro correspondiente ni figuraban en el padrón de autorización de pesca otorgada por la Dirección Regional de Pesquería de L oreto.

No existe infraestructura portuaria (muelle, cámaras frigoríficas e instalaciones) para realizar en forma eficiente el desembarco, transporte y comercialización del pescado. Esta última se hace a través de los mayoristas y venta directa al público.

Todos los dueños de las embarcaciones encuestadas declararon sobre el al to costo que representa la compra de motores, repuestos, paños de red, hilos de nylon, corchos, plomos y demás insumos necesarios para el equipamiento de una Unidad Económica de Pesca. Los costos aumentan significativamente por los fletes en el transporte fluvial o aéreo desde Lima o el extranjero, teniendo el agregado de los fuertes impuestos con los que se grava a este rubro (IGV e ISC). Por todo ello, los armadores pesqueros de la región de Loreto se encuentran en inferiores condiciones a las existentes en la zona costera.

De todas las embarcaciones encuestadas, sólo una presentaba el equipo de seguridad (chalecos salvavidas, extinguidores y botiquín de primeros auxilios).

L os factores que limitan el número de viajes anuales y la duración de una faena de pesca son climáticos-estacionales y de disponibilidad de insumos para implementar una U nidad E conómica de Pesca en forma adecuada. En la época de creciente de los ríos, el agua inunda grandes extensiones de la foresta amazónica, dificultándose la captura al disponer los peces de mayor área de dispersión y protección. Sucede todo lo contrario en época de vaciante, en donde la vulnerabilidad de los peces a las artes de pesca se incrementa al encontrarse confinados en áreas más reducidas, aumentando y disminuyendo, respectivamente, los días de pesca y por consiguiente el número de viajes. Otro factor importante para planificar con tiempo las salidas y los volúmenes de captura, es el abastecimiento constante de hielo. La producción de las 5 fábricas existentes en la zona de Iquitos, no cubre la demanda de la flota pesquera comercial, originando un atraso en las salidas y pérdidas económicas a los pescadores. 


\section{DISCUSION}

Pese a que no existe infraestructura adecuada, el puerto de Belén-Iquitos, continúa siendo el lugar frecuente de desembarque de las capturas provenientes de la flota pesquera comercial, motivados más que nada por la cercanía al principal mercado de la ciudad.

Se ha mencionado que sólo el $80 \%$ de las embarcaciones encuestadas se encuentran registradas en la Capitanía de Puerto y los restantes no figuraban ni en el padrón de autorización de pesca, razón por la cual podemos deducir que la información incluida en los cuadros estadísticos del sector referente al número total de embarcaciones y volúmenes de captura, no es completa.

El uso de hilo nylon polifilamento en la confección de redes, se ha generalizado en la A mazonía peruana. No se conoce experiencias en la utilización de redes con hilo monofilamento en esta región. Sin embargo, se sabe que en la parte brasilera, es frecuente por su mayor eficiencia y fácil transporte.

En la evolución histórica de la flota pesquera comercial, se observa que durante los años 1984-1985 la captura por unidad de esfuerzo (cpue) aumentó considerablemente. En este último año las capturas fueron del orden de las 3,429 t., con un esfuerzo de 103 embarcaciones. En el año siguiente, el volumen de captura disminuyó drásticamente a 2,932 t. y aumentó el número de embarcaciones a 111, el mayor esfuerzo desplegado en los años considerados. Schaefer (1953), citado por Chapman (1981), explica este comportamiento típico de una pesquería cuando dice "... a niveles de esfuerzo extremadamente altos, la producción declinará hacia la sobrepesca y a niveles intermedios de esfuerzo, la producción tenderá hacia el máximo...". Esta última afirmación queda demostrada en el año 1988, cuando con un esfuerzo intermedio (86 embarcaciones) se logra capturar el mayor volumen de peces (3,580 t.) en los años considerados.

La explicación del por qué el número de embarcaciones disminuye desde 111, en 1986, hasta 40 en 1989, la da Crutchfield, J., citado por Chapman (1981), al analizar los modelos de producción y estrategias de administración de la pesquería amazónica. Según la cita, este autor convierte los valores físicos en económicos, con el fin de ilustrar la relación entre el costo total de flota a varios niveles de esfuerzo y la ganancia total, y establece que cada embarcación adicional, totalmente equipada, aumenta los costos en una cantidad adicional igual. Mientras haya ganancias posibles, nuevas embarcaciones entrarán en la flota, hasta que los costos de operación e inversión de capital igualen las ganancias, produciéndose la quiebra o el retiro de muchas de ellas.

Contrariamente a lo que se pensaba, con respecto a la rentabilidad, las embarcaciones pesqueras pequeñas fueron las más eficientes. La poca capacidad instalada del cajón isotérmico de muchas de las embarcaciones mayores, podrían 
estar originando una subutilización de las mismas. Por otro lado, el aumento de los costos por concepto de depreciación, al elevarse significativamente la inversión en bienes de capital, disminuye proporcionalmente el índice de eficiencia económica, sin embargo, consideramos que una capacidad de bodega mayor que la tomada en el análisis (25 t.) incrementaría positivamente la rentabilidad de las embarcaciones denominadas grandes. Esta misma consideración podría darse para las pequeñas y medianas, cuyos volúmenes de carga para el cálculo fueron 8 y 12 t., respectivamente (C uadro 4).

\section{CONCLUSIONES Y RECOMENDACIONES}

La información disponible sobre la flota pesquera de Iquitos, en lo que se refiere al número de embarcaciones y volúmenes de captura es incompleta. Por otro lado, las unidades de pesca carecen del equipo básico de seguridad (chalecos salvavidas, extinguidores y botiquín de primeros auxilios).

Se recomienda mejorar el sistema de registro y control de las embarcaciones pesqueras existentes para un mejor ordenamiento y seguridad.

No existe infraestructura portuaria de pesca en la zona de desembarque y se observa un desabastecimiento constante de hielo.

Es necesario construir un terminal pesquero y equiparlo adecuadamente para facilitar el desembarque, manipuleo, conservación, transporte y comercialización del pescado.

La flota pesquera es relativamente joven (promedio de 6 años), considerando que el período de vida media de una embarcación es de 12 años.

Altos costos para implementar y mantener una unidad pesquera (motores, redes, repuestos, etc.).

Sería conveniente liberar los impuestos que de manera directa o indirecta incrementan los costos en el equipamiento de una U nidad Económica de Pesca. A dicionalmente, establecer línea de crédito para los armadores pesqueros con el fin de implementar mejoras o construir nuevas unidades.

Uso generalizado del hilo nylon tipo polifilamento para la confección 0 armado de redes.

Se recomienda efectuar un estudio técnico-económico, con el fin de analizar la factibilidad de usar redes con hilo tipo monofilamento.

Las embarcaciones pesqueras pequeñas tuvieron el mayor índice de rentabilidad.

Como una recomendación final y para complementar este trabajo, es necesario realizar un análisis más detallado sobre la eficiencia técnica económica de las embarcaciones que conforman la flota pesquera comercial de Iquitos, para determinar cuales son las características más adecuadas y estandarizadas por medio del diseño de una unidad de pesca típica. 


\section{BIBLIOGRAFIA}

CHAPMAN, D. 1981, Evaluación de capturas en el río A mazonas. IMARPE. Informe 81(11): 66-95.

CRUZ-TRINIDAD, A. 1990. The Use of Surplus-Y ield M odels in the Econornic A nalysis of a Fishery, Fishbyte 8(2): 20-24.

FA O. 1968. Embarcaciones Científicas y Buques de Investigación. Fisheries Report 29(3). 